\title{
A aprendizagem do português em Macau: uma pedagogia crítica
}

\author{
Custódio C. Martins (UMAC) ${ }^{1}$
}

Resumo: A história do ensino do português em Macau tem acompanhado, naturalmente, os seus vários momentos históricos e consequentes mudanças sociais e políticas. Partindo de uma perspectiva socio-histórica, discutimos o ensino do português e o ensino de línguas no contexto desse território, bem como delineamos algumas orientações que têm contribuído para a definição do contexto pedagógico até aos dias de hoje.

Palavras-chave: crítica pedagógica; ensino de português em Macau; pós-colonialismo.

\section{Introdução}

As áreas de investigação de aquisição de L2, assim como da Linguística Aplicada têm vindo a conferir alguma flexibilidade às suas agendas nas últimas décadas, incorporando conhecimentos de outras disciplinas, perspectivando as suas orientações de forma interdisciplinar, dando, por vezes, lugar a novas abordagens teóricas e criando, consequentemente, sub-áreas de investigação (MYLES, 2010), que também têm contribuído para que se faça uso de metodologias de pesquisa mais ecléticas e variadas (DÖRNYEI, 2009, p. 241-242). Assim, a análise da produção dos aprendentes em contextos L2 já não se circunscreve apenas a uma análise linguística pura, mas perspectiva-se agora a importância e o papel que variáveis não linguísticas podem ter na interpretação da variação ao nível do desempenho.

Como afirmam Kalaja e Barcelos (2003), variáveis como a motivação, as estratégias e estilos de aprendizagem, e mesmo as crenças têm vindo a fazer parte das agendas de investigação. Estes dados revestem-se de grande importância na medida em que se entende que o processo de aprendizagem de uma língua estrangeira/segunda (e até a primeira língua) não se constrói apenas com base em dados linguísticos. O contexto de aprendizagem pode ser também determinante para a forma como o processo de aquisição se desenrola (MARTINS, 2008), pelas suas características e pela forma como enquadra e perspectiva os conteúdos linguísticos.

No presente artigo, concentramo-nos no papel que o contexto sociopolítico/sociolinguístico pode ter no processo de aprendizagem de uma língua e cuja influência pode ser determinante em sala de aula. Nesse sentido,

\footnotetext{
${ }^{1}$ Professor Auziliar na Universidade de Macau nas áreas de Linguística Aplicada, Aquisição de
} Segundas Línguas e Psicolinguística. E-mail: fshcm@umac.mo 
partimos de uma breve análise do contexto socio-histórico de Macau, desde a sua fundação, enquanto espaço político e social, para simultaneamente nos debruçarmos com mais detalhe sobre a forma como este tem vindo a influenciar as políticas de língua até aos dias de hoje.

\section{O contexto}

Tal como muitos outros contextos socioeducativos e socioculturais no contexto asiático, Macau é multicultural e multilingue (TEIXEIRA e SILVA e MARTINS, 2011, p. 231; PINHARANDA NUNES, no prelo), situação e características que advêm do contexto histórico. Entre 1553 e 1555 já os primeiros portugueses haviam chegado à península de Macau e ao delta do Rio das Pérolas. Até ao estabelecimento oficial do território, administrado por Portugal, a península de Macau foi sendo povoada por gentes de várias proveniências:

The number of inhabitants of Macau throughout the centuries fluctuated according to political and military trends from all forces in the region. (PINHARANDA NUNES, no prelo, p. 6).

Compreende-se, portanto, que a conjuntura inicial de Macau era complexa, tanto em termos sociais como em termos linguísticos (BAXTER, 2009, p. 278) nos primórdios do seu estabelecimento, tendo continuado até aos dias de hoje (RODRIGUES, 2004, p. 4). Para Rodrigues, esta complexidade com raízes históricas contribuiu para que o papel da língua portuguesa tenha vindo a ser:

[...] regulada por condicionalismos históricos e sociopolíticos que se foram reflectindo no seu valor social e determinaram o seu uso. Introduzida no Território na época da expansão, a sua difusão foi favorecida pelas relações comerciais estabelecidas pelos portugueses com diferentes povos asiáticos, bem como pela acção evangelizadora dos missionários portugueses (2004, p. 10).

Rodrigues salienta a indissociabilidade que sempre houve em Macau entre discurso político e políticas de língua, traçando o perfil histórico de Macau desde que se tornou território administrado por Portugal até ao período de transferência de soberania em 1999. Nesse perfil são evidentes as marcas de uma identidade nacional que se quer afirmar "universalista" e "humanista", onde o entendimento e a tolerância são pilares constantes nos discursos e decisões políticas ao longo de décadas, ignorando muitas vezes o que Rodrigues designa como "amnésia organizada", em referência à expressão cunhada por Pina-Cabral (2002a, p. 127). Com o intuito da sedimentação de

34 fragmentum, N. 35, parte II. Laboratório Corpus: UFSM, Out./ Dez. 2012 
uma hegemonia política, mas também social, a língua serviu ao longo de décadas como "traço de união" (PINHEIRO, 1999) entre comunidades, mas, sobretudo, entre pontos históricos.

Ao olharmos para alguns dos discursos e trabalhos apresentados nos anos que precederam a transferência de soberania de Macau para a República Popular da China, apercebemo-nos da importância que o poder político atribuiu nesse período às questões da língua. Segundo a então Secretária de Estado da Educação do Governo de Portugal:

Hoje podemos perguntar aqui, em Macau, porquê, para quê aprender português. As respostas são muitas e diversas. São históricas, são afectivas e políticas. São razões do passado, do presente e do futuro. São também, claro, razões práticas de veículo de comunicação entre o Norte e o Sul (particularmente África e América Latina) e também em Macau, de ligação entre grandes países que procuram o contacto (BENAVENTE, 1997, p. 6).

As palavras da então Secretária de Estado resumem, de forma clara e precisa, a visão que o poder político tem tido quanto ao papel de Macau plataforma de entrecruzamento de culturas e de ponte de ligação com a grande China, sendo a língua o seu motor, mas também a forma como entende "a língua" - enquanto plataforma de ligação "afectiva" entre um tempo de glórias passadas e o desejo de glórias futuras, sedimentadas na construção de uma identidade nacional que se quer pós-colonialista, moderna, mas cuja expressão se encontra profundamente enraizada numa representação iconográfica ${ }^{2}$ e até mítica de uma ideia de identidade nacional de cariz universalista e humanista oriunda, inevitavelmente, de ideais coloniais ${ }^{3}$.

\section{Colonialismo, pós-colonialismo e ensino do português}

Um arreigada identidade nacional, fundada nos tais princípios universalistas e humanistas, comum em vários contextos colonialistas, atribuiu sempre à língua um papel preponderante de união, mas também de veículo de transmissão desses valores. A este respeito Rodrigues (2004, p. 29) vê nos discursos pré- e pós-transferência de soberania em Macau o perpetuar da visão colonialista que:

$\mathrm{Na}$ abusiva proclamação de uma identidade lusófona que reinventa o colonialismo "à portuguesa" das teorias luso-tropicalistas e imagina

2 Rodrigues (2004) em referência a um questionário realizado junto da população portuguesa relativo à simbologia expressiva da identidade nacional, dá-nos conta de que as imagens/símbolos do período das Descobertas são os mais salientes na memória colectiva.

${ }^{3}$ Cf. a este respeito, por exemplo, Sousa Santos (2001). 
quase que uma mega-nação lusófona - construída sobre coisas esquecidas umas e silenciadas outras - vemos o interesse na criação de relações privilegiadas com as ex-colónias, que compensem o lugar periférico que Portugal ocupa na cena europeia, servindo ao mesmo tempo os interesses económicos.

Para Rodrigues, este tipo de discurso esconde uma falsa realidade - a do conceito de Lusofonia, que a autora avalia de uma perspectiva crítica por considerar que se trata de uma visão de imperialismo linguístico, por dar pouco espaço às variedades linguísticas dos vários espaços "lusófonos", ao contrário do que vem acontecendo com a língua inglesa. Pelo menos a nível académico tem havido um reconhecimento de que o inglês não se restringe apenas a um padrão único e hegemónico, mas que diferentes variedades existentes passaram a ser plenamente reconhecidas.

$\mathrm{O}$ ensino da língua portuguesa em Macau, enquanto parte integrante do currículo, tem início na década de 70, mas foi nos anos 80, em ambiente pré-transferência de soberania, que a sua implementação foi mais forte e também mais dinâmica. $\mathrm{Na}$ sua generalidade, os professores que tinham a seu cargo o ensino da língua em Macau, numa fase inicial, eram, de formação de base, professores do que então se designava como ensino primário, e numa fase posterior, professores do ensino secundário recrutados a Portugal. No entanto, se alguns tinham formação específica no ensino de línguas estrangeiras, a grande maioria tinha formação nas mais diferentes áreas ${ }^{4}$.

O desenvolvimento do ensino do português em Macau, sobretudo na década de 80, teve na sua base conceções de carácter ideológico-político. Negociava-se por essa altura o acordo entre Portugal e a República Popular da China para a transferência de soberania. Até então pouco ou nada se tinha feito pelo ensino do português 5 .

O Portugal pós-revolução não soube ajustar-se às novas realidades e aos novos tempos, e a política de divulgação de língua, em consonância com a Europa em evolução constante, era praticamente inexistente. Foi sobretudo a experiência do ensino do português em África como língua "materna" que foi transportado para o contexto de Macau. Uma vez que Macau estava sob administração portuguesa, o ensino da língua, numa fase inicial, foi encarado no mesmo plano da língua materna. Ainda que tenha havido um esforço para alterar esta situação, a década de 80 não alterou os mitos que se tinham já instalado relativamente ao ensino/aprendizagem do português por alunos chineses. Estes baseavam-se, principalmente em dois vectores (1) nas diferenças culturais que se consideravam existir entre ambas as culturas, e se consideram, ainda hoje como fundamentais na estruturação de todo o ensino

${ }^{4}$ Cf. também a este respeito Rodrigues (2004, p. 226).

${ }^{5}$ Cf. Grosso (2007, p. 37) e também Rodrigues (2004).

36 fragmentum, N. 35, parte II. Laboratório Corpus: UFSM, Out./ Dez. 2012 
do português como língua estrangeira; (2) nas características específicas dos alunos chineses, dada a forte influência dos princípios confucionistas na educação e nas escolas do Território e da região ${ }^{6}$.

Estes aspetos tiveram, naturalmente, uma forte repercussão em Macau ao nível da implementação dos programas de ensino/aprendizagem do português. Se para alguns a multiculturalidade pode constituir uma mais-valia para o contexto educativo, para outros essa riqueza multilingue e multicultural pode apresentar dificuldades de vária ordem, nomeadamente ao nível da organização das experiências educativas. A perspectiva dominante em Macau, até aos dias de hoje, tem tido ecos nas afirmações de Domingues et al. (1997, p. 669) que no contexto de uma comunicação sobre os programas de língua, apresentada num Seminário sobre o ensino do Português como Língua Estrangeira referem:

Por outro lado, as equipas tiveram naturalmente em conta os princípios que pauteiam o ensino-aprendizagem de uma língua estrangeira com fins comunicativos, bem como o contexto de Macau já que a aprendizagem de uma língua estrangeira é condicionada pela forma como o aprendente adquire / adquiriu a língua materna e é esta que ao longo do processo de aprendizagem lhe vai servir como ponto de referência.

As autoras acrescentam ainda:

As abordagens de ensino do sistema chinês é diferente da abordagem do sistema de ensino português e é fundamental o conhecimento deste contexto na definição de estratégias de ensino e de actividades de aprendizagem dos alunos. Assim, na definição de estratégias de ensinoaprendizagem procurou-se conciliar estratégias mais comunicativas com as que o aluno chinês está habituado.

Numa perspectiva determinista, características culturais específicas têm sido apontadas por alguns investigadores como justificação para essas possíveis diferenças entre contextos de aprendizagem, mais especificamente no contraste entre Oriente e Ocidente. Esta posição determinista tem sido responsável pela construção de um sistema de crenças específico, sobretudo no que diz respeito à aprendizagem da língua portuguesa por aprendentes orientais.

Os contextos de ensino/aprendizagem asiáticos têm vindo a ser contrapostos aos contextos ocidentais, pelas diferenças culturais subjacentes a

6 Os princípios confucionistas da educação e em particular a sua influência ao nível do ensino/aprendizagem da língua estrangeira, nomeadamente do inglês, são revistos e analisados em Kellen (2002). 
cada (CHENG, 2000; XIE, 2009). Grosso (2007), por exemplo, defende a existência de um "método oriental", que caracteriza como (i) estruturado; (ii) com momentos pedagógicos claramente definidos; (iii) assume a imitação como a estratégia de aprendizagem por excelência. Este tipo de descrição dos contextos pedagógicos asiáticos tem as suas raízes, de acordo com vários investigadores no âmbito das ciências sociais, na herança confuciana destas sociedades 7 .

A este respeito, Rodrigues afirma:

Esta representação dos alunos chineses como "acanhados", "submissos" e presos a "velhas tradições" corresponde a um estereótipo cultural construído a partir das imagens orientalistas que o Ocidente em determinada época produziu sobre a China, e que a inexistência de investigação sobre os contextos de aprendizagem chineses, as suas crenças epistémicas e normas sociais, permitiu que se mantivesse até hoje (2004, p. 228).

A esta perspectiva de ensino de língua estava associada uma determinada cultura pedagógica que muitas vezes serviu como justificativa de uma falsa "ecologia cultural" do entendimento entre culturas. O que nos parece sempre faltou ao contexto sociocultural e também, por consequência, ao contexto educativo (por uma clara ausência de políticas) em Macau, foi uma consciência alargada e multifacetada de interculturalidade - característica inerente ao contexto de Macau. Kellen (2002, p. 236) reforça bem essa necessidade e importância:

In intercultural environments success depends on achieving the best and not the worst of both worlds. One has not to feel merely that one is constrained by two sets of rules but rather that one has a view into two different worlds, that one is able to tackle the difficult task of seeing those worlds from each others' point of view. The key to achieving such a goal will be in suspending one's disbelief for long enough to succeed in thinking in those other words which seem so alien to begin with.

A breve e sucinta análise da representação do ensino do português em Macau, sobretudo nas últimas décadas, demonstra que tem havido alguma dificuldade em conjugar esses "dois olhares" distintos, segundo a designação de Kellen, de modo a que eles se complementem e não se contraponham de forma quase irreversível.

${ }^{7}$ Autores como Ho et al (1995), Biggs (1999), Lee (1999) designam estas culturas como "Culturas de Herança Confuciana" (Confucian Heritage Cultures - CHC).

38 fragmentum, N. 35, parte II. Laboratório Corpus: UFSM, Out./ Dez. 2012 


\section{Considerações Finais}

Parece-nos estar no momento de refletir sobre o todo o processo de implementação do ensino do português em Macau, relativamente ao seu papel, aos seus objetivos e ao seu futuro, por ser uma questão que deve ser pensada de forma desarreigada do passado. Temos que ter bem presente que, como afirma Larsen-Freman (2003), é a perspectiva da língua que estiver inscrita nas nossas crenças, enquanto falantes dessa língua, que irá determinar o seu percurso didáctico-pedagógico, e consequentemente político. $\mathrm{O}$ ensino do português em Macau não pode estar preso a visões românticas do passado, sobretudo visões reificadas de cultura que continuam a considerar o "outro" segundo conceitos diferenciados, imaginários e estereotipados. O ensino do português em Macau deve tratar o contexto socioeducativo, bem como os seus intervenientes, como dinâmicos.

\section{Referências}

BAXTER, Alan, N. O Português em Macau: contacto e assimilação. In: CARVAlHO, Ana M. (org.). Português em Contacto. Madrid: Iberoamericana Vervuert, 2009, p. 277 - 313.

BENAVENTE, Ana. Discurso de Abertura do Seminário Internacional de Português como Língua Estrangeira. Macau, 2009 [1997].

BIGGS, John. Western misperceptions of the Confucian-heritage learning culture. David A. Watkins and John B. Biggs (Eds.) The Chinese Learner: Cultural, Psychological and Contextual Influences, CERC and ACER, University of Hong Kong, 1999.

CHENG, X. Asian students' reticence revisited. System, 28 (3), 435-446, 2000.

DOMINGUES, Edite et al. Os Programas de Língua Portuguesa como Língua Estrangeira Integrados na Reforma do Sistema Educativo de Macau. Actas do Seminário Internacional de Português como Língua Estrangeira, Universidade de Macau, Instituto Politécnico de Macau, Direcção dos Serviços de Educação e Juventude de Macau, Fundação Macau, 1997.

DÖRNYEI, Zoltán. The Psychology of the Language Learner - Individual Differences in Second Language Acquisition, Lawrence Erlbaum Associates, Publishers Mahwah, New Jersey, London, 2005.

GROSSO, Maria José dos Reis. O Discurso Metodológico do Ensino do Português em Macau a Falantes de Língua Materna Chinesa, Universidade de Macau, 2007.

HO, Judy et al. Breaking with Chinese Cultural Traditions: Learner Autonomy in English Language Teaching. System, v. 23, n. 2, p. 235-243, 1995. 
KALAJA, Paula et al. Researching Beliefs About SLA: A Critical Review. Paula Kalaja and Ana Maria Ferreira Barcelos (Eds.). Beliefs about SLA - New Research Approaches, Springer, 2003.

KELLEN, Christopher. Language and learning orthodoxy in the English classroom in China. Educational Philosophy and Theory 34, p. 223-237, 2002.

LARSEN-FREEMAN, Diane. Teaching Language: From Grammar to Grammaring. Pearson Publishers, 2003.

LEE, Wing On. On The Cultural Context for Chinese Learners: Conceptions of Learning in the Confucian Tradition. David A. Watkins and John B. Biggs (Eds.). The Chinese Learner: Cultural, Psychological, and Contextual influences, Hong Kong: CERC \& ACER, 1999.

MARTINS, C. C. A aquisição das noções de tempo e aspecto por aprendentes chineses de português como língua estrangeira. Tese de Doutoramento, Universidade de Macau, 2008.

MYLES, Florence. Research Timeline - The development of theories of second language acquisition. Language Teaching, 43:3, p. 320-332, Cambridge University Press, 2010.

PINA-CABRAL. J. Between China and Europe: person, culture and emotion in Macau. London: Continuum, 2002a.

PINHARANDA NUNES. Socio-Historical Factors Involved in Changes on the Creole Matrix of Makista (Routledge). (no prelo).

PINHEIRO, J. Língua Portuguesa: traço de união? A cooperação com os Países Africanos de Língua Oficial Portuguesa no âmbito da formação de professores. In: Actas do VIII Encontro da Associação das Universidades de Língua Portuguesa, Macau, 1998. Lisboa: Associação das Universidades de Língua Portuguesa, pp. 785-790, 1999.

RODRIGUES, Maria Helena da Fonseca de Oliveira. O Ensino do Português em Macau: Uma Abordagem Pós-Luso-Tropicalista. Tese de Doutoramento, Department of Hispanic Studies, School of Humanities, University of Birmingham, 2004.

SANTOS, B. S. Entre Prospero e Caliban: Colonialismo, pós-colonialismo e interidentidade. In: RAMALHO, M.I.; RIBEIRO, A.S. (orgs.). Entre Ser e Estar: Raízes, percursos e discursos da identidade. Porto: Afrontamento, p. 2385, 2001.

TEIXEIRA E SILVA et al. Intercultural Interaction: Teacher and Student Roles in the Classroom of Portuguese as A Foreign Language in Macau, China. In: PREISLER, Bent; KLITGARD, Ida; FABRICIUS, Anne H. (Eds.). Language and Learning in the International University - from English Uniformity to Diversity and Hybridity, Languages for Intercultural Communication and Education, Multilingual Matters, Bristol, Buffalo, Toronto, 2011.

40 fragmentum, N. 35, parte II. Laboratório Corpus: UFSM, Out./ Dez. 2012 
XIE, Xiaoyan. Why are students quiet? Looking at the Chinese context and beyond. ELT Journal, Volume 64/1, January, p. 10-20, Oxford University Press, 2009. 